\title{
A letter of reply to: Salehmohamed, M.R., Griffin, M., Branigan, T. et al. Patients treated with immunosuppressive steroids are less aware of sick day rules than those on endocrine replacement therapy and may be at greater risk of adrenal crisis. Ir J Med Sci (2017). doi:10.1007/s11845-017-1607-y
}

\author{
L. Griffin ${ }^{1}$ (I)
}

Received: 15 June 2017 / Accepted: 4 July 2017 / Published online: 25 July 2017

(C) Royal Academy of Medicine in Ireland 2017

Dear Sir or Madam,

I write to agree with Salemohammed et al. [1] that the sick day rules for corticosteroid cover must be understood by other non-endocrine specialties in the interests of patient safety.

In current dermatology practice, oral steroids are used for inflammatory conditions such as vasculitis, giant cell arteritis, pyoderma gangrenosum, bullous pemphigoid, and pemphigus vulgaris. Although there is a trend towards early introduction of steroid-sparing agents, patients can remain on gradually reducing doses of corticosteroids for months. Patients tend to be older and have multiple co-morbidities. Dermatologists commonly address the idea of bone and gastroprotection at steroid initiation but the steroid sick day rules are inadequately considered.

Reviewing the British Association of Dermatology patient information leaflet, which is commonly used by Irish dermatologists when initiating long-term oral medications, there is mention of "avoiding sudden withdrawal" and the "need to wear MedicAlert bracelet" but the sick day rules are not specified! (http://www.bad.org.uk/shared/get-file.ashx?id= 112\&itemtype=document) The UK document should be updated and Dermatology, in addition to all specialities, must adopt a steroid sick day rules policy to routine practice.

Kind Regards,

Dr. Laoise Griffin

Dermatology Registrar, University Hospital Limerick and General Practitioner

MICGP, MRCPUK, M Med Sc. (Sports and Ex Med), P. Dip Clin Education

\section{Reference}

1. Salehmohamed MR, Griffin M, Branigan T et al (2017) Patients treated with immunosuppressive steroids are less aware of sick day rules than those on endocrine replacement therapy and may be at greater risk of adrenal crisis. Ir J Med Sci. doi:10.1007/ s11845-017-1607-y
L. Griffin

laoisegriffin@gmail.com

1 University Hospital Limerick, Limerick, Ireland 